\title{
Biopsia neuroendoscópica. Revisión de la literatura y experiencia en 31 pacientes
}

M. Domínguez-Páez; M. Puch-Ramírez; S. Rodríguez-Barceló; J.M. Medina-Imbroda; L. Romero-Moreno; G. Ibáñez-Botella; B. Ros-López y M.A. Arráez-Sánchez

Servicio de Neurocirugía. Hospital Regional Universitario Carlos Haya. Málaga.

Resumen

Objetivos. Presentar nuestra experiencia en el manejo endoscópico de tumores intraventriculares, analizando la efectividad diagnóstica de la biopsia, y comparar los resultados obtenidos con los datos publicados en la literatura.

Material y métodos. Presentamos una serie de 31 pacientes con edades comprendidas entre los 7 meses y los 77 años, diagnosticados de proceso expansivo intra $\mathrm{y} / \mathrm{o}$ periventricular, sólido $\mathrm{y} / \mathrm{o}$ quístico, e intervenidos quirúrgicamente en nuestro servicio entre 2003 y 2010 para la realización de una biopsia neuroendoscópica. Analizamos la técnica utilizada, el resultado anatomopatológico obtenido, el manejo de la hidrocefalia asociada, la frecuencia de complicaciones del procedimiento y la actitud terapéutica posterior.

Resultados. Se realizaron 32 procedimientos neuroendoscópicos, obteniéndose muestra para biopsia en 28 de ellos, con resultado positivo en $25 \mathbf{( 7 8 \%}$ de éxito por procedimiento y $89 \%$ de éxito por biopsia). El diagnóstico histológico más frecuente fue de astrocitoma grado II. 30 pacientes presentaban hidrocefalia asociada practicándose una ventriculostomía premamilar (VPM) en 19 casos (éxito en 14 casos, un 73.7\%); además, se realizó una septostomía en 12 pacientes (en 3 casos asociada a VPM y en 9 casos seguida de derivación ventrículo-peritoneal). En 3 casos el procedimiento endoscópico fue asistido con neuronavegación. Durante la cirugía y el postoperatorio se contabilizaron las siguientes complicaciones: hemorragia intraventricular en cuatro pacientes (en dos casos se produjo éxitus), crisis comiciales en dos pacientes, focalidad neurológica en tres casos (signo de Parinaud, paresia transitoria del III par craneal y hemiparesia asociada a parálisis del III par), y fístula de líquido cefalorraquídeo con infección meníngea en un caso. 19 pacientes recibieron tratamiento posterior (quirúrgico en 1 caso, radiocirugía estereotáxica en 2 casos, radioterapia en 8

Recibido: 25-03-10. Aceptado: 23-02-11 casos, quimioterapia en 5 casos y quimio-radioterapia en 3 casos).

Conclusiones. El abordaje endoscópico de las lesiones intra $\mathrm{y} / \mathrm{o}$ periventriculares presenta un alto rendimiento diagnóstico, permitiendo además en muchos casos tratar la hidrocefalia asociada con un único procedimiento, por lo que podría ser el tratamiento de elección en lesiones consideradas no resecables. Aunque no está exento de complicaciones graves, la morbimortalidad podría ser inferior a la de los abordajes microquirúrgicos convencionales.

PALABRAS CLAVE. Biopsia endoscópica. Tumores ventriculares.

Neuroendoscopic biopsy. Experience in 31 patients and literature review

\section{Summary}

Objective. To describe our experience with the endoscopic management of intraventricular tumors, analyzing biopsy effectiveness, and to compare our results with those obtained from an extensive literature review.

Materials and methods. Between 2003 and 2010, 31 patients aged between 7 months and 77 years, diagnosed of solid and/or cystic intra and/or periventricular tumors, underwent neuroendoscopic biopsy. We analyze operative technique, pathological result, management of associated hydrocephalus, rate of complications and postoperative technique.

Results. 32 endoscopic procedures were done and biopsy was successfully performed in 28 cases, with

Abreviaturas. AP: astrocitoma pilocítico. APM: astrocitoma pilomixoide. DVE: drenaje ventricular externo. DVP: derivación ventrículo peritoneal. GBM: glioblastoma multiforme. HTIC: hipertensión intracraneal. HIV: hemorragia intraventricular. LCR: líquido cefalorraquídeo. N: número. VPM: ventriculostomía premamilar endoscópica. 
positive histological result in 25 of them ( $78 \%$ success rate per procedure and $89 \%$ success rate per biopsy). Most frequent pathological diagnosis was grade II astrocytoma. 30 patients had associated hydrocephalus that required endoscopic third ventriculostomy (19 cases, with $73.7 \%$ success rate) and/or septostomy (12 patients, 3 associated with ventriculostomy and 9 with ventriculo-peritoneal shunt). Frameless neuronavigation was used in three selected cases. During the surgery and the postoperative period the following complications appeared: intraventricular hemorrhage in four cases (two of them died), seizures in two patients, new neurological findings in three cases (Parinaud's sign, transient palsy of third cranial nerve and hemiparesis associated with palsy of third cranial nerve), and cerebrospinal fluid leak and infection in one case. 19 patients received subsequent treatment (microsurgical resection in 1, radiosurgery in 2, radiotherapy in 8, chemotherapy in 5 and chemo-radiotherapy in 3 ).

Conclusions. Endoscopic management of intraventricular and/or periventricular brain tumors is effective, and allow diagnostic biopsy and simultaneous treatment of the associated hydrocephalus in many cases. So, it could be the treatment of choice in those tumors that are not suitable for microsurgical resection. Although this technique is not exempt of serious complications, morbimortality could be lower than conventional microsurgical approach.

KEY WORDS. Endoscopic biopsy. Ventricular tumors.

\section{Introducción}

La endoscopia es una técnica que se utiliza en neurocirugía desde los años 20 cuando Dandy y Mixter empezaron a usar cistoscopios y ureteroscopios para abordar los ventrículos ${ }^{4,13}$. La neuroendoscopia moderna comienza en la década de los 70 con la aparición de nuevos instrumentos rígidos y flexibles. La publicación de la primera serie de endoscopias para biopsia de tumores intraventriculares la realiza Fukushima en 1978, con un éxito del $52 \%$ en 21 pacientes $^{7}$. Actualmente es una técnica efectiva en el manejo de los tumores ventriculares y de la hidrocefalia asociada, describiéndose diferentes variantes en cuanto a la localización del trépano, número de abordajes, tipo de endoscopio, indicación de septostomía y orden de la biopsia y de la ventriculostomía premamilar $(\mathrm{VPM})^{1,2,5,12,14,16,21,23,24}$. Aportamos nuestra experiencia de los últimos 7 años en neuroendoscopia aplicada al manejo de lesiones expansivas intra y/o periventriculares, y comparamos los resultados con lo descrito en la literatura hasta el momento.
Material y métodos

\section{Pacientes}

31 pacientes con el diagnóstico de tumor intra $\mathrm{y} / \mathrm{o}$ periventricular sólido y/o quístico fueron intervenidos en nuestro servicio entre los años 2003 y 2010 para la realización de una biopsia mediante neuroendoscopia, descartándose en un primer momento cirugía resectiva debido a la imposibilidad técnica o a que no se consideró necesaria por el aspecto radiológico de la lesión. La recogida de datos clínicos, radiológicos y quirúrgicos de estos 31 pacientes fue realizada de forma retrospectiva.

La relación hombre/mujer fue de 17/14 y la edad media de 39 años (rango comprendido entre los 7 meses y los 77 años). 25 tumores eran sólidos, 4 sólido-quísticos y 2 quísticos en RMN, siendo la localización más frecuente la región posterior del tercer ventrículo con 13 casos, seguido del tercer ventrículo anterior con 10 , región periventricular con 6 y ventrículos laterales con 2 casos. En el momento del diagnóstico la hidrocefalia estaba presente en el 96' $8 \%$ de los casos. La presentación clínica más frecuente fue en forma de hipertensión intracraneal (HTIC), asociada a focalidad neurológica en 6 casos.

\section{Técnica endoscópica}

Todos los procedimientos se realizaron bajo anestesia general, con la cabeza en posición neutra, flexionada y fijada con craneostato de Mayfield. Se utilizaron dos modelos de endoscopio rígido de $8 \mathrm{~mm}$ de diámetro, un canal de trabajo y óptica de $0^{\circ}$. Se realizó un solo trépano frontal a 10-13 cm del nasion y a $3 \mathrm{~cm}$ de la línea media, derecho siempre que permitiese tratar la hidrocefalia y tomar las muestras de biopsia. Generalmente se situó el trépano a 10 $\mathrm{cm}$ del nasion para la biopsia de lesiones de mitad posterior del tercer ventrículo, lo que a su vez otorga un ángulo adecuado para realizar la VPM sin lesionar el pilar anterior del fórnix. La estabilidad del endoscopio se consiguió con la sujeción manual del ayudante. Durante el procedimiento se utilizó bomba de irrigación con suero tipo Ringer para lavar puntualmente. Los procedimientos realizados con el endoscopio fueron la toma de biopsias de la lesión y los relacionados con la restauración de las vías de LCR: VPM y/o septostomía. La VPM se llevó a cabo mediante apertura mecánica y dilatación posterior del estoma, utilizando un balón Fogarty no específico para endoscopia, del número 4 en adultos y del 3 en pacientes pediátricos. Se utilizó la navegación en tres casos seleccionados.

\section{Resultados}

En los 31 pacientes se practicaron 32 procedimientos endoscópicos con el fin de obtener un diagnóstico histológico. Se obtuvo material tumoral en 28 procedi- 


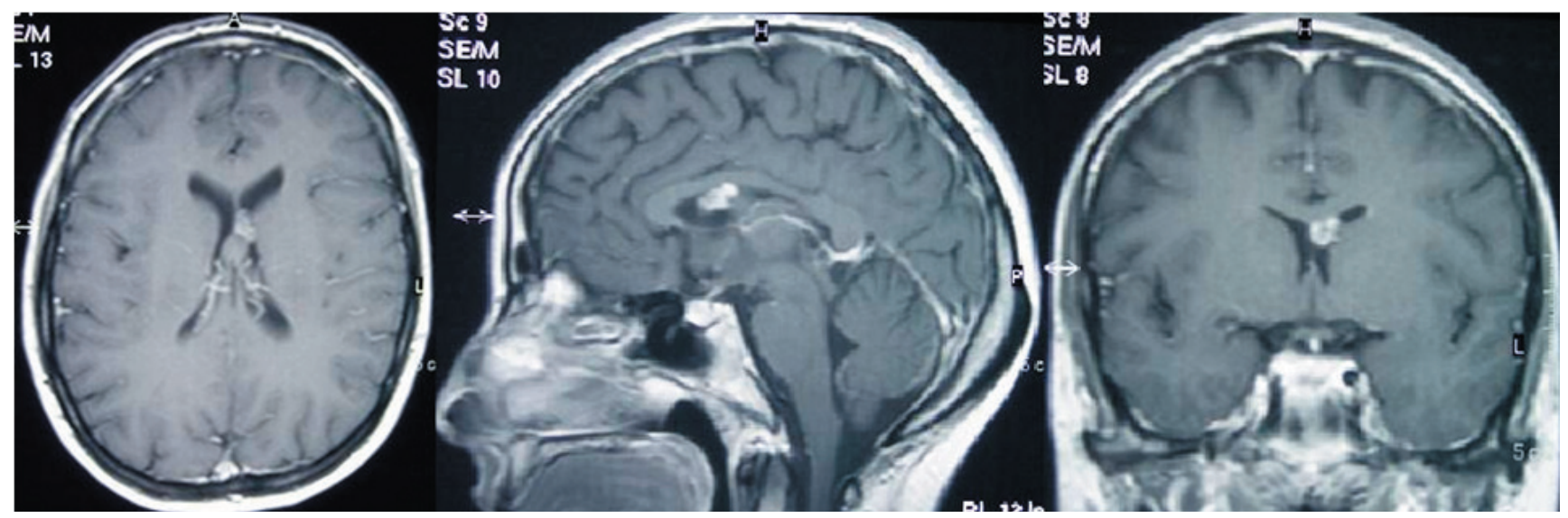

Ejemplo 1. Caso 18. Mujer de 34 años con cuadro de cefalea inespecifica. En la RM se aprecia lesión nodular en ventrículo lateral izquierdo con infiltración de septum pellucidum y cuerpo calloso. Se realiza biopsia ampliada guiada con neuronavegación. El diagnóstico histológico es de ependimoma de bajo grado. La paciente recibe radiocirugía posterior en 2007 y actualmente se encuentra en seguimiento.

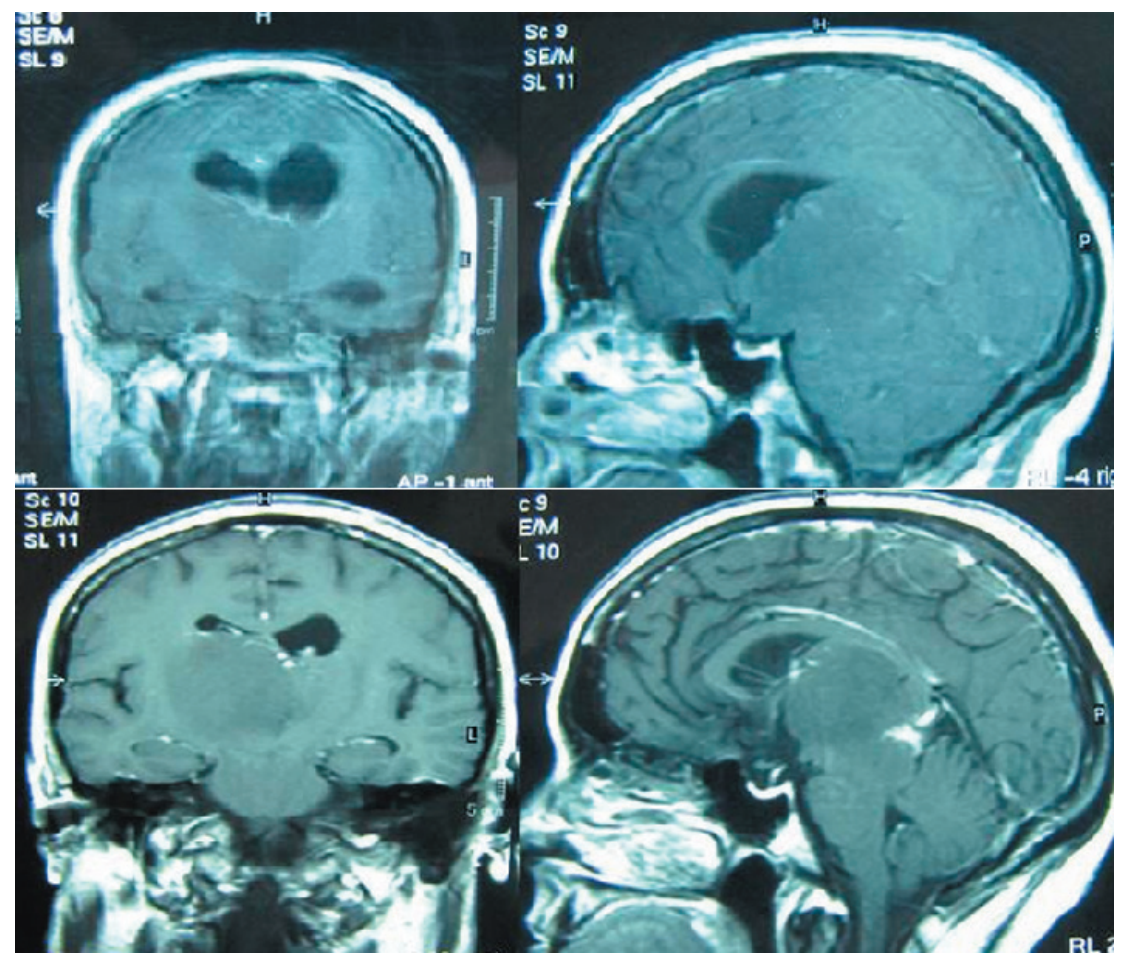

Ejemplo 2. Caso 1. Mujer de 47 años con cuadro de cefalea progresiva. En la RMse aprecia lesión periventricular difusa que colapsa el tercer ventrículo provocando una hidrocefaliabiventricular (fotos superiores). Se realiza ventriculostomía premamilar y biopsia endoscópica, consiguiéndose resolver la hidrocefalia (fotos inferiores). El diagnóstico histológico es de astrocitoma grado II por lo que la paciente recibe tratamiento complementario con radioterapia.

mientos con un rango de 1 a 7 muestras (media de 4). De acuerdo con los criterios de Depreitere, en 25 procedimientos las biopsias fueron diagnósticas (78\% de éxito por procedimiento y $89 \%$ de éxito por biopsia), 2 mostraron tejido patológico no identificable y una tejido no interpretable. La mayor rentabilidad diagnóstica se obtuvo con las lesiones localizadas en los ventrículos laterales y mitad anterior del tercer ventrículo $(100 \%)$, seguidas de las de mitad posterior del III ventrículo (84.6\%) y las periventriculares $(33 \%)$. El diagnóstico anatomopatológico más frecuente fue el de astrocitoma grado II con 8 casos (32\%), de los cuales 4 fueron astrocitomas pilomixoides (APM), seguido de 4 casos de craneofaringioma, 2 casos de astrocitoma pilocítico (AP) y glioblastoma multiforme (GBM), y un solo caso de pineoblastoma, meduloblastoma, disgerminoma, tumor del seno endodérmico, ependimoma de bajo grado, astrocitoma grado III, adenoma de hipófisis productor de ACTH, metástasis de adenocarcinoma de próstata y linfoma no Hodgkin de células grandes B. En los pacientes 26,27 y 28 la toma de muestra no fue posible, en el primer caso por una hemorragia que imposibilitó la canalización ventricular y, en los otros dos casos, por ser consi- 


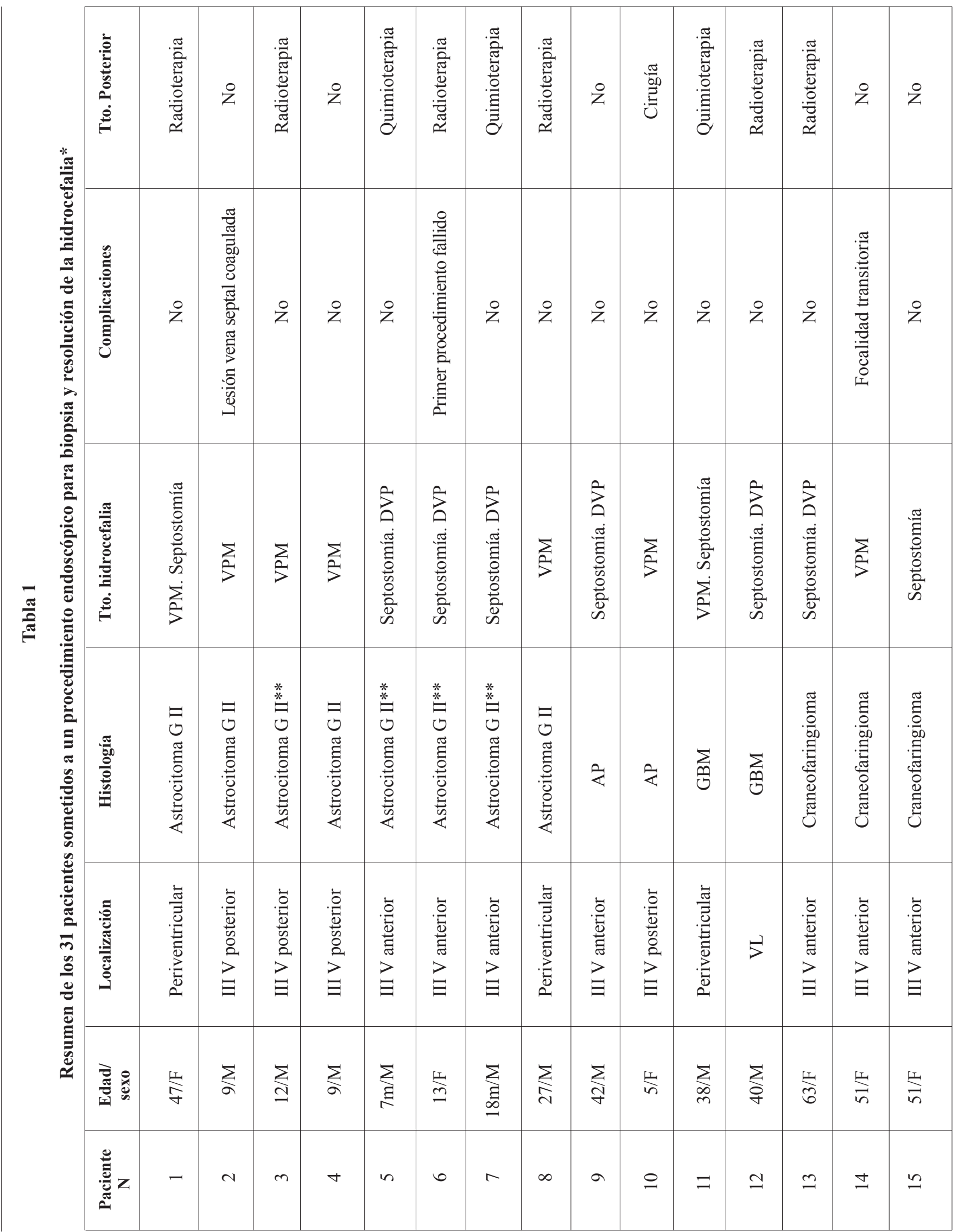




\begin{tabular}{|c|c|c|c|c|c|c|c|c|c|c|c|c|c|c|c|c|}
\hline 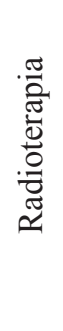 & 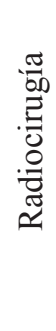 & 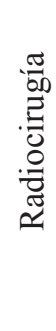 & 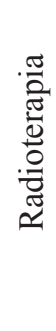 & Z & 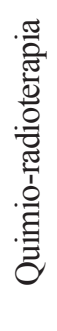 & 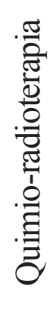 & 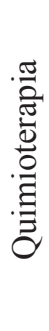 & 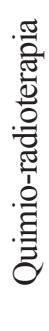 & 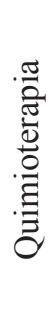 & $\stackrel{\circ}{z}$ & : & ż & $z_{z}$ & $\stackrel{\circ}{z}$ & $\stackrel{\circ}{z}$ & 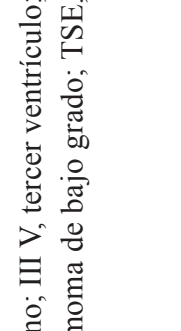 \\
\hline$z$ & 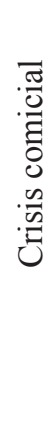 & z & 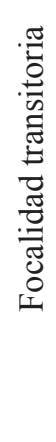 & 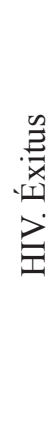 & 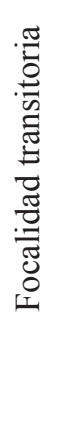 & $\stackrel{\circ}{z}$ & $\stackrel{\circ}{Z}$ & 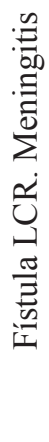 & z & 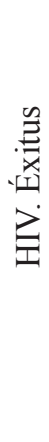 & $\stackrel{0}{z}$ & $z_{z}$ & $z_{z}$ & $z_{z}$ & 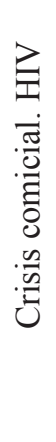 & 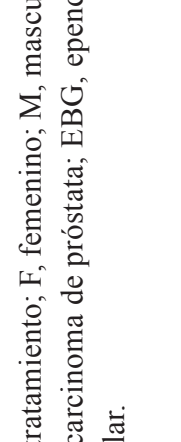 \\
\hline 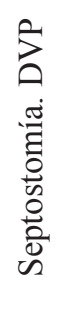 & $\sum_{>}$ & $\stackrel{2}{z}$ & $\sum_{>}$ & 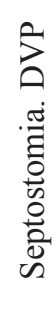 & $\sum_{j}$ & 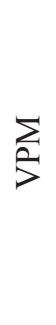 & 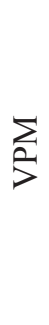 & $\sum_{j}^{\infty}$ & 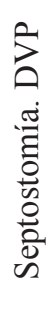 & $\stackrel{\circ}{z}$ & 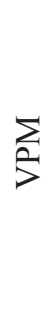 & 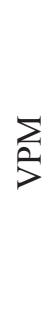 & 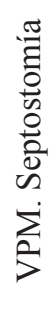 & 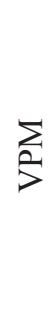 & $\sum_{>}$ & 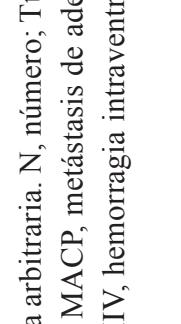 \\
\hline 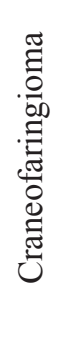 & $\underset{\Sigma}{\Sigma}$ & $\begin{array}{l}0 \\
M \\
\text { M }\end{array}$ & 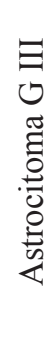 & 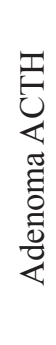 & $\begin{array}{l}\text { In } \\
\mathscr{H}\end{array}$ & 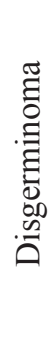 & 兄 & 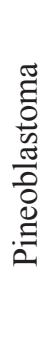 & 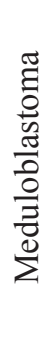 & $\begin{array}{l}\frac{\pi}{\sqrt[n]{n}} \\
\frac{2}{0} \\
\frac{0}{0} \\
0 \\
z\end{array}$ & $\begin{array}{l}. \frac{\pi}{0} \\
0 \\
0 \\
0 \\
0 \\
0 \\
z\end{array}$ & $\begin{array}{l}\frac{\pi}{0} \\
\frac{0}{0} \\
0 \\
0 \\
0\end{array}$ & 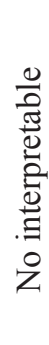 & 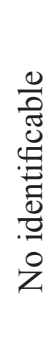 & 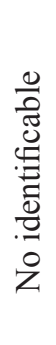 & 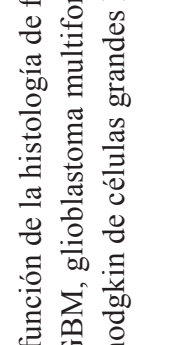 \\
\hline 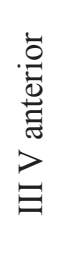 & 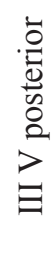 & $\$$ & 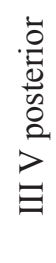 & 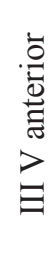 & 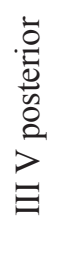 & 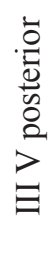 & 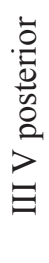 & 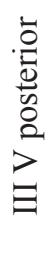 & 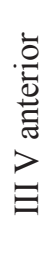 & 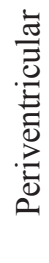 & 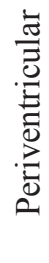 & 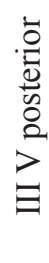 & 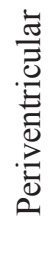 & 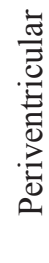 & 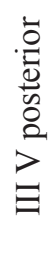 & 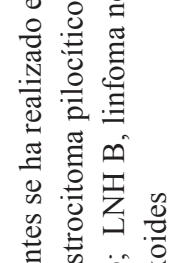 \\
\hline 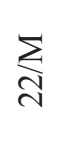 & $\underset{⿱}{\underset{\nabla}{*}}$ & $\underset{⿱ 乛 龰}{\stackrel{f}{f}}$ & $\underset{i n}{\sum}$ & $\sum_{n}$ & 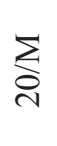 & $\sum_{n}$ & $\underset{n}{\stackrel{n}{n}}$ & $\underset{\infty}{\infty}$ & $\sum_{m}$ & $\underset{6}{\frac{1}{6}}$ & $\underset{\sim}{\stackrel{ \pm}{\sim}}$ & $\underset{N}{\sum}$ & $\underset{⿱}{\stackrel{1}{\leqslant}}$ & $\underset{⿱}{\mathbb{N}}$ & $\underset{i n}{\sum_{i n}}$ & 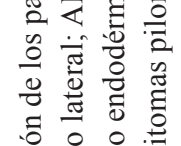 \\
\hline 으 & I & $\stackrel{\infty}{-}$ & 9 & 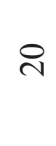 & $\vec{\sim}$ & $\widetilde{N}$ & $\tilde{\sim}$ & $\stackrel{\sim}{\sim}$ & $\stackrel{2}{\sim}$ & $\stackrel{?}{\sim}$ & $\grave{\imath}$ & $\stackrel{\infty}{\sim}$ & ते & in & $\vec{m}$ & 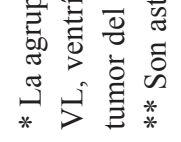 \\
\hline
\end{tabular}


derados de alto riesgo (ausencia de impronta de la lesión en la superficie intraventricular y localización periacueductal respectivamente).

30 pacientes presentaban hidrocefalia asociada que precisó VPM en 19 casos (éxito inicial en 16 casos, un 84.2\%; produciéndose un fallo de la VPM a los 4 y 13 meses en los casos número 22 y 1 respectivamente); además, se realizó una septostomía en 12 pacientes (en 3 casos asociada a VPM y en 9 casos seguida de DVP). Se consiguió diagnóstico histológico y resolver de forma conjunta la hidrocefalia con VPM en 11/19 pacientes (58\%). La neuronavegación se aplicó en los casos 18 y 31 con éxito, mientras que en el caso 8 se abandonó por el desplazamiento de las estructuras cerebrales tras la salida de LCR.

Durante la cirugía y el postoperatorio se produjeron las siguientes complicaciones: hemorragia intraventricular (HIV) en cuatro casos (en el paciente 2 se lesionó de forma mecánica la vena septal durante la movilización del endoscopio para abordar la región posterior del tercer ventrículo, controlándose la hemorragia intraoperatoriamente con coagulación bipolar; el paciente 20 sufrió una HIV durante el postoperatorio; el paciente 26 sufrió una hemorragia durante la maniobra de canalización ventricular obligando al abandono del procedimiento endoscópico; en el paciente 31 la hemorragia se produjo durante la retirada del endoscopio, sin identificarse el punto sangrante. Los pacientes 20, 26 y 31 precisaron un DVE, con fallecimiento en los dos primeros casos por la hemorragia y resolución completa en el tercer caso), crisis comiciales en los pacientes 17 y 31 durante el postoperatorio inmediato, focalidad neurológica en tres casos (signo de Parinaud en el paciente 19, paresia transitoria de tercer par craneal en el 21 y hemiparesia asociada a parálisis del tercer par craneal en el 14), y fístula de LCR e infección meníngea en el caso 24. El paciente 6 requirió dos procedimientos endoscópicos ya que en el primero no se consiguió canalizar el ventrículo. 19 pacientes recibieron tratamiento posterior definitivo y/o paliativo (cirugía en 1, radiocirugía en 2, radioterapia en 8 , quimioterapia en 5 y quimio-radioterapia en 3). Se perdió el seguimiento del paciente 27 , y el 15 falleció por progresión de la enfermedad (ver tabla 1).

\section{Discusión}

Indicación. En la actualidad la neuroendoscopia en el manejo de las lesiones centroencefálicas se considera un abordaje mínimamente invasivo con las ventajas de reducir el tiempo de hospitalización, de retorno a la vida cotidiana y de impacto estético ${ }^{1}$, siendo además un procedimiento más corto ${ }^{8}$ y barato ${ }^{9}$ que el abordaje quirúrgico clásico. Puesto que no todas las lesiones intra-periventriculares se benefician de la resección microquirúrgica ${ }^{2,8,21,23,24}$, y dado que ésta implica una morbimortalidad que oscila entre el
$5 \%$ y el $15 \%$-debida fundamentalmente a la disección y retracción de importantes estructuras cerebrales-, la biopsia neuroendoscópica emerge como una alternativa diagnóstica que permite decidir el tratamiento de elección en este tipo de situación ${ }^{2,22,24}$. Estirpes histológicas como los tumores germinales, los linfomas o la enfermedad metastásica diseminada, o localizaciones como el diencéfalo, no son habitualmente subsidiarias de tratamiento microquirúrgico. Así, la neuroendoscopia adquiere especial relevancia en las masas pineales, donde la heterogeneidad histológica es elevada (20-37\% de tumores de células germinales, $22-27 \%$ de tumores del tejido pineal, $24-28 \%$ de gliomas y $12-32 \%$ de otras histologías) ${ }^{2}$; en los niños donde la incidencia de tumores germinales es alta ${ }^{21} \mathrm{y}$ en las lesiones quísticas, que pueden ser aspiradas e, incluso, resecadas ${ }^{1}$. Adicionalmente, la endoscopia presenta la ventaja de posibilitar el tratamiento de la hidrocefalia asociada, presente en el $73.7 \%$ de nuestros casos y en aproximadamente el $90 \%$ de las lesiones intra-periventriculares referidas en la literatura ${ }^{1,2,5,6,8,12,18,21-24}$, con un éxito terapéutico similar al descrito en la VPM para el tratamiento de la estenosis del acueducto de Silvio y que puede variar entre el 60-100\% en función de la serie $e^{2,14,18,23}$. Además permite obtener muestras de LCR para citología y marcadores tumorales ${ }^{6,12} \mathrm{e}$ identificar diseminaciones radiológicamente no visibles ${ }^{15}$. Nuestra serie, en la que únicamente un paciente $(3.2 \%)$ requirió tratamiento quirúrgico posterior, parece confirmar los resultados de la literatura, posicionando la biopsia neuroendoscópica como una excelente opción terapéutica en lesiones centroencefálicas con hidrocefalia en las que la microcirugía y/o la estereotaxia no sean consideradas como primera opción de tratamiento ${ }^{2,6}$.

Técnica. Una vez indicada la neuroendoscopia se deben tener en cuenta una serie de detalles técnicos. Se recomienda que el trépano tenga forma cónica para aumentar el grado de maniobrabilidad del endoscopio ${ }^{1,21}$, aunque en nuestra serie sólo se han realizado trépanos con broca estándar, y que esté localizado aproximadamente a un centímetro precoronal y tres centímetros de la línea media. En caso de que se vaya a abordar una lesión de la mitad posterior del tercer ventrículo se recomienda hacer el trépano algo más anterior que en una VPM convencional para evitar retracciones corticales $^{1,14,21}$; en nuestra experiencia consideramos que localizarlo a $10 \mathrm{~cm}$ del nasion es suficiente para la realización de la biopsia y la VPM sin lesionar el pilar anterior del fórnix. En caso de lesiones localizadas en los ventrículos laterales se suele preferir el abordaje por el hemisferio no dominante. Las lesiones hipotalámicas suelen abordarse a través del hemisferio controlateral. Si el paciente presenta una asimetría ventricular es preferible penetrar por el más dilatado $^{21}$. Aunque la mayoría de los autores realiza un abordaje único se han descrito variantes como la propuesta 
por $\mathrm{Chernov}^{2}$, que defiende la realización de un abordaje bilateral, lo que facilitaría el control de un sangrado intraventricular, o la propuesta por Yurtseven ${ }^{24}$, que aboga por un abordaje doble homolateral (uno para la realización de la VPM y otro para la biopsia de lesiones de la mitad posterior del tercer ventrículo) con el fin de minimizar la retracción de las estructuras cerebrales. Hasta el momento tanto los sistemas de endoscopio rígidos como flexibles han mostrado su eficacia sin diferencias significativas, especialmente a la hora de abordar lesiones de la mitad posterior del tercer ventrículo ${ }^{2}$. La ventaja de los sistemas flexibles radica en su maniobrabilidad, ya que minimizan el daño a los tejidos centroencefálicos durante las diferente maniobras y permiten seleccionar mejor la zona de biopsia; los sistemas rígidos, por el contrario, posibilitan obtener una muestra mayor y ofrecen mejor visualización ${ }^{2,12,14}$. Autores como Oi usan al inicio un sistema rígido para explorar seguido de uno flexible para realizar la VPM y la biopsia ${ }^{16}$. A veces es necesario realizar una septostomía, como por ejemplo para alcanzar el sistema ventricular controlateral o intentar evitar un catéter en "Y" en aquellas lesiones de tercio anterior del tercer ventrículo con hidrocefalia; algunos autores prefieren realizarla de forma sistemática con el fin de explorar posibles siembras metastásicas, descartar hematomas en el sistema ventricular controlateral o prevenir una posible hidrocefalia por obstrucción del foramen de Monro $^{2}$. El uso de irrigación continua puede facilitar la eliminación de restos tumorales/tisulares que floten en el LCR y minimizar la hipotensión de líquido, que a veces se asocia a colecciones extraaxiales, aunque se deben extremar las precauciones para no producir un incremento de presión lesivo para el paciente ${ }^{2}$. Nosotros empleamos solución Ringer de forma puntual, por ejemplo para detener sangrados de escasa cuantía durante el procedimiento. Una vez visualizada la lesión se recomienda la exploración de la misma para identificar la zona de adhesión al parénquima adyacente y obtener muestras de biopsia de diferentes zonas, ya que algunas neoplasias son heterogéneas ${ }^{24}$. Es muy importante una coagulación minuciosa de la zona biopsiada $^{2,5,21}$. Aunque algunos autores recomiendan coagular de forma previa a la toma de muestra para minimizar el riesgo de sangrado ${ }^{2,24}$, es posible que se produzca con ello un artefacto térmico ${ }^{21}$. Si la lesión es periventricular se recomienda realizar la biopsia en la zona subependimaria y tomar el mínimo número de muestras posible para evitar un sangrado incontrolable ${ }^{2,12}$. Se ha asociado a un fracaso diagnóstico de la biopsia un volumen de muestra escaso y que la lesión se localice en la fosa posterior ${ }^{5,12,23}$. Existe la posibilidad de asistir la endoscopia con algún sistema de imagen que facilite el procedimiento, destacando la estereotaxia (permite una trayectoria idónea a la lesión) $)^{21}$, la neuronavegación (contribuye a la seguridad del procedimiento, especialmente si se necesita canalizar un ventrículo sin hidrocefalia $)^{2,12}$ y la ecografía en caso de lactantes ${ }^{5}$. Para nosotros la neuronavegación es un procedimiento útil que ayuda a orientar el endoscopio dentro de la anatomía intraventricular. Sin embargo, el desplazamiento de las estructuras cerebrales tras la pérdida de LCR puede llevar a confusión. Si existe una indicación de VPM junto la biopsia hay disparidad de criterios en la literatura a la hora de establecer qué procedimiento realizar en primer lugar. Se considera a favor de la biopsia el evitar que pase sangre al espacio subaracnoideo, con el consiguiente riesgo de hidrocefalia, permitir aumentar el volumen de la cavidad ventricular con irrigación para manipular mejor la lesión y limitar el riesgo de diseminación ${ }^{2,24}$. A favor de la VPM, evitar que un sangrado tumoral obligue a abandonar el procedimiento sin haber tratado la hidrocefalia ${ }^{5,12,14,21}$; es más, para algunos autores el objetivo principal del procedimiento endoscópico es éste, quedando en segundo lugar la realización de la biopsia ${ }^{12,23}$. Nosotros preferimos realizar en primer lugar la VPM. Si el paciente es portador de DVP algunos autores recomiendan la retirada del catéter proximal ya que se postula que aumenta el porcentaje de fallo de la VPM, el riesgo de infección y la diseminación tumoral $^{2}$. Es importante asegurar la fenestración de la membrana de Liliquiest durante la $\mathrm{VPM}^{2,6}$.

Éxito del procedimiento. En las diferentes series revisadas desde 1999 encontramos 332 biopsias endoscópicas realizadas, con un éxito diagnóstico en 289 de ellas (87\% por biopsia obtenida) $)^{1,2,5,6,8,12,14,21-24}$. Referente al tratamiento de la hidrocefalia consigue resolverse en 109 de 132 procedimientos (éxito del $82.6 \%$ ) $^{2,5,8,14,21-23}$. Estas cifras son equiparables a las obtenidas en el tratamiento de la estenosis del acueducto de Silvio ${ }^{2,14,18,23}$ (ver tabla 2). En la serie que presentamos el éxito diagnóstico por biopsia es del $89 \%$ y la tasa de éxito de la VPM es del $73.7 \%$.

Estereotaxia vs endoscopia. Según Gaab y Schroeder la endoscopia es superior en el manejo de lesiones intraventriculares y pineales ${ }^{8,20}$. Si se compara la revisión de 54 casos de Yamini ${ }^{23}$ de biopsias endoscópicas pineales con la de Kreth ${ }^{11}$ de 106 casos de biopsias estereotáxicas, el éxito diagnóstico de la biopsia estereotáxica es mayor: $89 \%$ vs $97 \%$ respectivamente. Sin embargo, la endoscopia tiene una serie de ventajas que se basan en la visualización directa, destacando la elección de la zona a biopsiar y la posibilidad de controlar complicaciones como la hemorragia $^{2,8,23}$. Además, la estereotaxia no permite tratar la hidrocefalia en el mismo acto quirúrgico ${ }^{2,8,14,24}$, habiéndose descrito el error por migración de la lesión al drenar líquido una vez se punza el ventrículo ${ }^{24}$. En lo referente a la realización de la VPM, la endoscopia ha mostrado ser técnicamente más factible que la estereotaxia $(94.1 \%$ vs $87.2 \%$ respectivamente) $)^{3}$. 
Tabla 2

Resumen de la revisión bibliográfica

\begin{tabular}{|l|l|c|}
\hline Trabajo & Éxito por biopsia & Éxito por ventriculostomía y/o stent \\
\hline Badie, B.1 & $6 / 47(98 \%)$ & -- \\
\hline Chernov, M.F.2 & $23 / 23(100 \%)$ & $22 / 23(95 \%)$ \\
\hline Depreitere, B.5 & $22 / 31(71 \%)$ & $9 / 14(64 \%)$ \\
\hline O'Brien, D.F.14 & $25 / 33(76 \%)$ & $28 / 41(68 \%)$ \\
\hline Fiorindi, A.6 & $16 / 23(70 \%)$ & --- \\
\hline Gaab, M.R.8 & $5 / 6(83 \%)$ & $6 / 6(100 \%)$ \\
\hline Macarthur, N.12 & $17 / 28(61 \%)$ & --- \\
\hline Souweidane, M.M.21 & $23 / 23(100 \%)$ & $22 / 22(100 \%)$ \\
\hline Tirakotai, W.22 & $46 / 46(100 \%)$ & $20 / 20(100 \%)$ \\
\hline Yamini, B.23 & $48 / 54(89 \%)$ & $2 / 6(33 \%)$ \\
\hline Yurtseven, T.24 & $18 / 18(100 \%)$ & --- \\
\hline Éxito global & $289 / 332(87 \%)$ & $109 / 132\left(82{ }^{\prime} 6 \%\right)$ \\
\hline
\end{tabular}

$V P M$ vs $D V P$. A pesar de que la VPM no es una técnica exenta de riesgos (mortalidad del $0-1 \%$ y morbilidad del $6-16.7 \%$ en función de la serie) $)^{2,19,20}$, parece ofrecer una serie de ventajas respecto la DVP, ya que elimina el riesgo de diseminación peritoneal, evita tener que incluir la infección del sistema derivativo en el diagnóstico diferencial de la fiebre en pacientes con tratamiento quimioterápico ${ }^{12}, \mathrm{y}$ obvia las complicaciones valvulares (riesgo de disfunción del $25-40 \%$ durante el primer año, y de un $4-5 \%$ anual en los siguientes; así como un riesgo de infección del $5-10 \%^{2,14}$, aunque en algunas series se describe hasta un $\left.20 \%{ }^{23}\right)$.

Limitaciones. Se ha descrito como una de las principales limitaciones de la neuroendoscopia el inadecuado control de las hemorragias cuantiosas ${ }^{6}$. Se postula que técnicas como la coagulación con láser podrían ayudar a solventar esta complicación ${ }^{12,17}$. Aún no se ha conseguido determinar si la diseminación leptomeníngea de algunas neoplasias puede estar favorecida por la técnica endoscópica o si es parte de la evolución natural de la enfermedad ${ }^{2,16}$. Otra posible limitación es que se requiere de un patólogo con la suficiente pericia como para poder interpretar de forma correcta las pequeñas muestras tumorales ${ }^{21}$. A veces el tipo de tumor puede determinar el éxito diagnóstico del procedimiento a pesar de una correcta técnica, ya que las lesiones consideradas "duras" pueden dificultar enormemente la realización de la biopsia ${ }^{14,21}$.

Complicaciones. La complicación más temible y problemática es el sangrado ${ }^{2,8}$, con un riesgo de hemorragia significativa (aquélla que tiene repercusión clínica u obliga a abandonar el procedimiento) del $3.6 \%$ en la serie de 55 procedimientos de Luther ${ }^{9}$. En caso de hemorragias pequeñas el control suele ser adecuado con irrigación, mientras que los sangrados cuantiosos requieren de coagulación con bipolar y, si la hemostasia no es suficiente, suspender el procedimiento y colocar un DVE ${ }^{2,8,9}$. Para minimizar el riesgo la planificación preoperatoria con angio-RM, angio-TC y/o arteriografía es de gran ayuda, así como los hallazgos intraoperatorios; abandonar el procedimiento en lesiones de aspecto muy vascular puede ser una opción adecuada $^{2}$, ya que podemos enfrentarnos a situaciones como la descrita por Ishihara en la que un aneurisma del top de la basilar simulaba una neoplasia de tercer ventrículo ${ }^{10}$. En la revisión de la literatura realizada la incidencia de hemorragia significativa por procedimiento es del $2.33 \%$, no habiéndose descrito ningún caso de mortalidad atribuible al procedimiento ${ }^{2,5,6,8,9,14,21-24}$. En nuestra serie la tasa de hemorragia clínicamente significativa y de mortalidad por procedimiento es del $9.4 \%$ y del $6.2 \%$ respectivamente. Dentro de las diferentes complicaciones que pueden aparecer destaca la fístula de LCR, la focalidad neurológica transitoria (siendo la oftalmoparesia la más frecuente), la 
meningitis, la hiponatremia y el coma por edema del tronco encefálico ${ }^{5,8,12,14,22,23}$.

\section{Conclusiones}

El abordaje endoscópico de las lesiones intraventriculares y/o periventriculares con fines diagnósticos es un procedimiento efectivo que permite tratar la hidrocefalia en muchos de estos pacientes, y obtener un diagnóstico en el mismo acto quirúrgico que posibilite un posterior tratamiento de la lesión. La neuroendoscopia permite la toma de biopsias en estas regiones anatómicas bajo visión directa, pudiendo elegir de forma más adecuada el área óptima para la biopsia. La tasa de morbimortalidad del procedimiento es baja, siendo la hemorragia la complicación más temible.

\section{Bibliografía}

1. Badie, B., Brooks, N., Souweidane, M.M.: Endoscopic and minimally invasive microsurgical approaches for treating brain tumor patients. J Neurooncol 2004; 69: 209-219.

2. Chernov, M.F., Kamikawa, S., Yamane, F., Ishihara, S., Kubo, O., Hori, T.: Neurofiberscopic biopsy of tumors of the pineal region and posterior third ventricle: indications, technique, complications, and results. Neurosurgery 2006; 59: 267-277.

3. Cinalli, G., Sainte-Rose, C., Chumas, P., et al.: Failure of third ventriculostomy in the treatment of aqueductal stenosis in children. Neurosurgical Focus 1999; 6: E3.

4. Dandy, W.: Treatment of non-capsulated brain tumors by extensive resection of contiguous braintissue. Bull Jhons Hpkins Hosp 1992; 33: 189-190.

5. Depreitere, B., Dasi, N., Rutka, J., Dirks, P., Drake, J.: Endoscopic biopsy for intraventricular tumors in children. J Neurosurg 2007; 106: 340-346.

6. Fiorindi, A., Longatti, P.: A restricted neuroendoscopic approach for pathological diagnosis of intraventricular and paraventricular tumours. Acta Neurochir (Wien) 2008; 150: 1235-1239

7. Fukushima, T: Endoscopic biopsy of intraventricular tumors with the use of a ventriculofiberscope. Neurosurgery 1978; 2: 110-113.

8. Gaab, M.R., Schroeder, M.D.: Neuroendoscopic approach to intraventricular lesions. Neurosurg Focus 1999; 6: E5.

9. Luther, N., Cohen, A., Souweidane, M.M.: Hemorrhagic sequelae from intracranial neuroendoscopic procedures for intraventricular tumors. Neurosurg Focus 2005; 19: E9.

10. Ishihara, S., Kamikawa, S., Suzuki, C., et al.: Neuroendoscopic identification of a basilar artery tip aneurysm in the third ventricle. Case illustration. J Neurosurg 2002; 96: 1138.

11. Kreth, F.W., Schätz, C.R., Pagenstecher, A., Faist, M., Volk, B., Ostertag, C.B.: Stereotactic management of lesions of the pineal region. Neurosurgery 1996; 39: 280-289.

12. Macarthur, D.C, Buxton, N., Punt J, Vloeberghs, M.,
Robertson, I.J.: The role of neuroendoscopy in the management of brain tumours. Br J Neurosurg 2002; 16: 465-470.

13. Mixter, W.: Ventriculoscopy and puncture of the floor of the tirad ventricle. Bost Med Surg J 1923; 188: 277-278.

14. O'Brien, D.F., Hayhurst, C., Pizer, B., Mallucci, C.L.: Outcomes in patients undergoing single-trajectory endoscopic third ventriculostomy and endoscopic biopsy for midline tumors presenting with obstructive hydrocephalus. J Neurosurg 2006; 105: 219-226.

15. Oi, S., Kamio, M., Joki, T., Abe, T.: Neuroendoscopic anatomy and surgery in pineal region tumors: role of neuroendoscopic procedure in the 'minimally-invasive preferential' management. J Neurooncol 2001; 54: 277-286.

16. Oi, S., Shibata, M., Tominaga, J., et al.: Efficacy of neuroendoscopic procedures in minimally invasive preferential management of pineal region tumors: a prospective study. J Neurosurg 2000; 93: 245-253.

17. Oka, K., Kin, Y., Go, Y., et al.: Neuroendoscopic approach to tectal tumors: a consecutive series. Neurosurg Focus 1999; 15: E14.

18. Ray, P., Jallo, G.I., Kim, R.Y., et al.: Endoscopic third ventriculostomy for tumor-related hydrocephalus in a pediatric population. Neurosurg Focus 2005; 19: E8.

19. Sainte-Rose, C., Cinalli, G., Roux, F.E., et al.: Management of hydrocephalus in pediatric patients with posterior fossa tumors: the role of endoscopic third ventriculostomy. $\mathrm{J}$ Neurosurg 2001; 95: 791-797.

20. Schroeder, H.W., Niendorf, W.R., Gabb, M.R.: Complications of endoscopic third ventriculostomy. J Neurosurg 2002, 96: 1032-1040.

21. Souweidane, M.M.: Endoscopic management of pediatric brain tumors. Neurosurg Focus 2005; 18 (6A): E1.

22. Tirakotai, W., Hellwig, D., Bertalanffy H., Riegel, T.: The role of neuroendoscopy in the management of solid or solid-cystic intra- and periventricular tumours. Childs Nerv Syst 2007; 23: 653-658.

23. Yamini, B., Refai, D., Rubin, C.M., Frim, D.M.: Initial endoscopic management of pineal region tumors and associated hydrocephalus: clinical series and literature review. J Neurosurg 2004; 100: 437-441.

24. Yurtseven, T., Ersahin, Y., Demirtas, E., Mutluer, S.: Neuroendoscopic biopsy for intraventricular tumors. Minim Invasive Neurosurg 2003; 46: 293-299.

Domínguez-Páez, M.; Puch-Ramírez, M.; Rodríguez-Barceló, S.; Medina-Imbroda, J.M.; Romero-Moreno, L.; IbáñezBotella, G. ; Ros-López, B.; Arráez-Sánchez, M.A.:Biopsia neuroendoscópica. Revisión de la literatura y experiencia en 31 pacientes. Neurocirugía 2011; 22: 419-428.

Correspondencia: Servicio de Neurocirugía. Hospital Regional Universitario Carlos Haya. Avenida Carlos Haya, s/n. 29010. Málaga. Emaillenin_@hotmail.com 
Comentario al trabajo Biopsia neuroendoscópica. Revisión de la literatura y experiencia en 31 pacientes de $\mathrm{M}$. Domínguez-Páez y cols.

En este trabajo Domínguez-Páez y colaboradores muestran su experiencia en el manejo endoscópico de 31 pacientes con lesiones intra y paraventriculares, con unos resultados superponibles a los descritos en la literatura. La neuroendoscopia es una técnica mínimamente invasiva que permite, en un único procedimiento, tratar la hidrocefalia que prácticamente de forma constante presentan estos pacientes y obtener muestras de biopsia que posibiliten el diagnóstico histológico. Sin embargo, la morbilidad de la misma -tal y como muestra este trabajo, con 9 complicaciones en 32 procedimientos (28.1\%) y una tasa de hemorragia clínicamente significativa del $9.4 \%$ - no es desdeñable, como tampoco lo es su potencial mortalidad (6.2\%). Es por ello que deben extremarse las precauciones a la hora de sentar su indicación y tener en consideración que el procedimiento combinado de biopsia y ventriculostomía/septostomía endoscópica, al igual que casi cualquier técnica quirúrgica con una especial curva de aprendizaje, debe ser realizado por cirujanos con un adecuado entrenamiento en neuroendoscopia. Por otro lado, las alternativas en forma de derivación licuoral y abordaje microquirúrgico o biopsia estereotáxica no ofrecen un perfil de riesgo-beneficio claramente superior, sobre todo teniendo en cuenta las complicaciones a largo plazo de los sistemas valvulares, de todos conocidas.

Desde el punto de vista técnico, coincidimos con los autores en la secuencia de tratamiento endoscópico, favoreciendo la realización de la ventriculostomía/ septostomía en la primera parte de la intervención, ya que consideramos la hidrocefalia como el problema fundamental a solucionar y la biopsia como una potencial fuente de complicaciones en forma de sangrado. Al igual que Domínguez-Páez y cols, también intentamos reali- zar el procedimiento con un único agujero de trépano, para lo que nos resulta de especial ayuda la navegación, ya que nos permite seleccionar un punto de entrada óptimo que posibilite proceder con las dos trayectorias de la cirugía minimizando el riesgo de lesión estructural. En tumores paraventriculares es también útil para determinar una trayectoria ortogonal al lugar donde la lesión se encuentre en contacto directo con el epéndimo o aflorando a través del mismo. Sin embargo, una vez la trayectoria ha sido fijada y se ha penetrado en el sistema ventricular, la navegación no aporta grandes beneficios durante la cirugía, si exceptuamos aquellos casos aislados de anatomía ventricular muy distorsionada o con ventrículos de tamaño reducido, en los que puede ayudar a la orientación del cirujano.

\section{Bibliografía}

1. Ahmad, F., Sandberg, D.I.: Endoscopic management of intraventricular brain tumors in pediatric patients: a review of indications, techniques, and outcomes. J Child Neurol 2010; 25: 359-367.

2. Ahn, E.S., Goumnerova, L.: Endoscopic biopsy of brain tumors in children: diagnostic success and utility in guiding treatment strategies. J Neurosurg Pediatr 2010; 5: 255-262.

3. Naftel, R.P., Shannon, C.N., Reed, G.T., et al.: Smallventricle neuroendoscopy for pediatric brain tumor management. J Neurosurg Pediatr 2011; 7: 104-110.

4. Song, J.H., Kong, D.S., Seol, H.J., Shin, H.J.: Transventricular biopsy of brain tumor without hydrocephalus using neuroendoscopy with navigation. J Korean Neurosurg Soc 2010; 47: 415-419. 